\title{
Some Properties of Alexandrov Topologies
}

Yong Chan Kim ${ }^{1}$ and Young Sun $\mathrm{Kim}^{2}$

${ }^{1}$ Department of Mathematics, Gangneung-Wonju National University, Gangneung 201-702, Korea

${ }^{2}$ Department of Applied Mathematics, Pai Chai University, Daejeon 302-735, Korea
Received: Jul. 9, 2014

Revised : Sep. 20, 2014

Accepted: Sep. 22, 2014

Correspondence to: Young Sun Kim (yskim@pcu.ac.kr)

(CThe Korean Institute of Intelligent Systems

(c) This is an Open Access article distributed under the terms of the Creative Commons Attribution Non-Commercial License (http://creativecommons.org/licenses/ by-nc/3.0/) which permits unrestricted noncommercial use, distribution, and reproduction in any medium, provided the original work is properly cited.

\begin{abstract}
Alexandrov topologies are the topologies induced by relations. This paper addresses the properties of Alexandrov topologies as the extensions of strong topologies and strong cotopologies in complete residuated lattices. With the concepts of Zhang's completeness, the notions are discussed as extensions of interior and closure operators in a sense as Pawlak's the rough set theory. It is shown that interior operators are meet preserving maps and closure operators are join preserving maps in the perspective of Zhang's definition.
\end{abstract}

Keywords: Complete residuated lattices, Alexandrov topologies, Fuzzy partially ordered set, Meet and join

\section{Introduction}

Pawlak [1,2] introduced the rough set theory as a formal tool to deal with imprecision and uncertainty in the data analysis. Hájek [3] introduced a complete residuated lattice which is an algebraic structure for many valued logic. By using the concepts of lower and upper approximation operators, information systems and decision rules are investigated in complete residuated lattices [3-7]. Zhang and Fan [8] and Zhang et al. [9] introduced the fuzzy complete lattice which is defined by join and meet on fuzzy partially ordered sets. Alexandrov topologies [7, 10-12] were introduced the extensions of fuzzy topology and strong topology [13].

In this paper, we investigate the properties of Alexandrov topologies as the extensions of strong topologies and strong cotopologies in complete residuated lattices. Moreover, we study the notions as extensions of interior and closure operators. We give their examples.

Definition 1.1. [3, 4] An algebra $(L, \wedge, \vee, \odot, \rightarrow, \perp, \top)$ is called a complete residuated lattice if it satisfies the following conditions:

(C1) $L=(L, \leq, \vee, \wedge, \perp, \top)$ is a complete lattice with the greatest element $\top$ and the least element $\perp$;

(C2) $(L, \odot, \top)$ is a commutative monoid;

(C3) $x \odot y \leq z$ iff $x \leq y \rightarrow z$ for $x, y, z \in L$.

In this paper, we assume $\left(L, \wedge, \vee, \odot, \rightarrow,{ }^{*} \perp, \top\right)$ is a complete residuated lattice with a negation; i.e., $x^{* *}=x$. For $\alpha \in L, A, \top_{x} \in L^{X},(\alpha \rightarrow A)(x)=\alpha \rightarrow A(x), \quad(\alpha \odot A)(x)=$ $\alpha \odot A(x)$ and $\top_{x}(x)=\top, \top_{x}(x)=\perp$, otherwise.

Lemma 1.2. [3, 4] For each $x, y, z, x_{i}, y_{i} \in L$, the following properties hold.

(1) If $y \leq z$, then $x \odot y \leq x \odot z$.

(2) If $y \leq z$, then $x \rightarrow y \leq x \rightarrow z$ and $z \rightarrow x \leq y \rightarrow x$. 
(3) $x \rightarrow y=\top$ iff $x \leq y$.

(4) $x \rightarrow \top=\top$ and $\top \rightarrow x=x$.

(5) $x \odot y \leq x \wedge y$.

(6) $x \odot\left(\bigvee_{i \in \Gamma} y_{i}\right)=\bigvee_{i \in \Gamma}\left(x \odot y_{i}\right)$

(7) $x \rightarrow\left(\bigwedge_{i \in \Gamma} y_{i}\right)=\bigwedge_{i \in \Gamma}\left(x \rightarrow y_{i}\right)$ and $\left(\bigvee_{i \in \Gamma} x_{i}\right) \rightarrow y=$ $\bigwedge_{i \in \Gamma}\left(x_{i} \rightarrow y\right)$.

(8) $\bigvee_{i \in \Gamma} x_{i} \rightarrow \bigvee_{i \in \Gamma} y_{i} \geq \bigwedge_{i \in \Gamma}\left(x_{i} \rightarrow y_{i}\right)$ and $\bigwedge_{i \in \Gamma} x_{i} \rightarrow$ $\bigwedge_{i \in \Gamma} y_{i} \geq \bigwedge_{i \in \Gamma}\left(x_{i} \rightarrow y_{i}\right)$.

(9) $(x \rightarrow y) \odot x \leq y$ and $(y \rightarrow z) \odot(x \rightarrow y) \leq(x \rightarrow z)$.

(10) $x \rightarrow y \leq(y \rightarrow z) \rightarrow(x \rightarrow z)$ and $x \rightarrow y \leq(z \rightarrow$ $x) \rightarrow(z \rightarrow y)$.

(11) $\bigwedge_{i \in \Gamma} x_{i}^{*}=\left(\bigvee_{i \in \Gamma} x_{i}\right)^{*}$ and $\bigvee_{i \in \Gamma} x_{i}^{*}=\left(\bigwedge_{i \in \Gamma} x_{i}\right)^{*}$

(12) $(x \odot y) \rightarrow z=x \rightarrow(y \rightarrow z)=y \rightarrow(x \rightarrow z)$ and $(x \odot y)^{*}=x \rightarrow y^{*}$.

(13) $x^{*} \rightarrow y^{*}=y \rightarrow x$ and $(x \rightarrow y)^{*}=x \odot y^{*}$.

(14) $y \rightarrow z \leq x \odot y \rightarrow x \odot z$.

(15) $x \rightarrow y \odot z \geq(x \rightarrow y) \odot z$ and $(x \rightarrow y) \rightarrow z \geq$ $x \odot(y \rightarrow z)$.

Definition 1.3. $[7,10,12,13]$ A subset $\tau \subset L^{X}$ is called an Alexandrov topology if it satisfies:

(T1) $\perp_{X}, \top_{X} \in \tau$ where $\top_{X}(x)=\top$ and $\perp_{X}(x)=\perp$ for $x \in X$.

(T2) If $A_{i} \in \tau$ for $i \in \Gamma, \bigvee_{i \in \Gamma} A_{i}, \bigwedge_{i \in \Gamma} A_{i} \in \tau$.

(T3) $\alpha \odot A \in \tau$ for all $\alpha \in L$ and $A \in \tau$.

(T4) $\alpha \rightarrow A \in \tau$ for all $\alpha \in L$ and $A \in \tau$.

A subset $\tau \subset L^{X}$ satisfying (T1), (T3) and (T4) is called a strong topology if it satisfies:

(ST) If $A_{i} \in \tau$ for $i \in \Gamma, \bigvee_{i \in \Gamma} A_{i}, \wedge_{i \in \Lambda} A_{i} \in \tau$ for each finite index $\Lambda \subset \Gamma$.

A subset $\tau \subset L^{X}$ satisfying (T1), (T3) and (T4) is called a strong cotopology if it satisfies:

(SC) If $A_{i} \in \tau$ for $i \in \Gamma, \bigwedge_{i \in \Gamma} A_{i}, \vee_{i \in \Lambda} A_{i} \in \tau$ for each finite index $\Lambda \subset \Gamma$.

Remark 1.4. Each Alexandrov topology is both strong topology and strong cotopology.

Definition 1.5. $[8,9]$ Let $X$ be a set. A function $e_{X}: X \times X \rightarrow$ $L$ is called:

(E1) reflexive if $e_{X}(x, x)=\top$ for all $x \in X$,

(E2) transitive if $e_{X}(x, y) \odot e_{X}(y, z) \leq e_{X}(x, z)$, for all $x, y, z \in X$,

(E3) if $e_{X}(x, y)=e_{X}(y, x)=\top$, then $x=y$.

If $e$ satisfies (E1) and (E2), $\left(X, e_{X}\right)$ is a fuzzy preordered set. If $e$ satisfies (E1), (E2) and (E3), $\left(X, e_{X}\right)$ is a fuzzy partially ordered set.
Example 1.6. (1) We define a function $e_{L^{X}}: L^{X} \times L^{X} \rightarrow L$ as $e_{L^{X}}(A, B)=\bigwedge_{x \in X}(A(x) \rightarrow B(x))$. Then $\left(L^{X}, e_{L^{X}}\right)$ is a fuzzy partially ordered set from Lemma 1.2 (8).

(2) Let $\tau$ be an Alexandrov topology. We define a function $e_{\tau}: \tau \times \tau \rightarrow L$ as $e_{\tau}(A, B)=\bigwedge_{x \in X}(A(x) \rightarrow B(x))$. Then $\left(\tau, e_{\tau}\right)$ is a fuzzy partially ordered set.

Definition 1.7. $[8,9]$ Let $\left(X, e_{X}\right)$ be a fuzzy partially ordered set and $A \in L^{X}$.

(1) A point $x_{0}$ is called a join of $A$, denoted by $x_{0}=\sqcup A$, if it satisfies

(J1) $A(x) \leq e_{X}\left(x, x_{0}\right)$,

(J2) $\bigwedge_{x \in X}\left(A(x) \rightarrow e_{X}(x, y)\right) \leq e_{X}\left(x_{0}, y\right)$.

A point $x_{1}$ is called a meet of $A$, denoted by $x_{1}=\sqcap A$, if it satisfies

(M1) $A(x) \leq e_{X}\left(x_{1}, x\right)$,

(M2) $\bigwedge_{x \in X}\left(A(x) \rightarrow e_{X}(y, x)\right) \leq e_{X}\left(y, x_{1}\right)$.

Remark 1.8. $[8,9]$ Let $\left(X, e_{X}\right)$ be a fuzzy partially ordered set and $A \in L^{X}$.

(1) $x_{0}$ is a join of $A$ iff

$$
\bigwedge_{x \in X}\left(A(x) \rightarrow e_{X}(x, y)\right)=e_{X}\left(x_{0}, y\right) .
$$

(2) $x_{1}$ is a meet of $A$ iff $\bigwedge_{x \in X}\left(A(x) \rightarrow e_{X}(y, x)\right)=$ $e_{X}\left(y, x_{1}\right)$.

(3) If $x_{0}$ is a join of $A$, then it is unique because $e_{X}\left(x_{0}, y\right)=$ $e_{X}\left(y_{0}, y\right)$ for all $y \in X$, put $y=x_{0}$ or $y=y_{0}$, then $e_{X}\left(x_{0}, y_{0}\right)$ $=e_{X}\left(y_{0}, x_{0}\right)=\top$ implies $x_{0}=y_{0}$. Similarly, if a meet of $A$ exist, then it is unique.

Remark 1.9. $[8,9]$ Let $\left(L^{X}, e_{L^{X}}\right)$ be a fuzzy partially ordered and $\Phi \in L^{L^{X}}$.

(1) Since

$$
\begin{aligned}
\bigwedge_{A \in L^{X}}\left(\Phi(A) \rightarrow e_{L^{X}}(A, B)\right) & =e_{L^{x}}\left(\bigvee_{A \in L^{X}}(\Phi(A) \odot A), B\right) \\
& =e_{L^{X}}(\sqcup \Phi, B),
\end{aligned}
$$

then $\sqcup \Phi=\bigvee_{A \in L^{X}}(\Phi(A) \odot A)$.

(2) We have $\sqcap \Phi=\bigwedge_{A \in L^{X}}(\Phi(A) \rightarrow A)$ because

$$
\begin{aligned}
& \bigwedge_{A \in L^{X}}\left(\Phi(A) \rightarrow e_{L^{X}}(B, A)\right. \\
& =\bigwedge_{A \in L^{X}} e_{L^{X}}(B,(\Phi(A) \rightarrow A)) \\
& =e_{L^{X}}\left(B, \bigwedge_{A \in L^{X}}(\Phi(A) \rightarrow A)\right) .
\end{aligned}
$$

\section{Some Properties of Alexandrov Topologies}

Theorem 2.1. (1) A subset $\tau \subset L^{X}$ is an Alexandrov topology on $X$ iff for each $\Phi: \tau \rightarrow L, \sqcup \Phi \in \tau$ and $\sqcap \Phi \in \tau$. 
(2) $\tau$ is an Alexandrov topology on $X$ iff $\tau^{*}=\left\{A^{*} \in L^{X}\right.$ $A \in \tau\}$ is an Alexandrov topology on $X$.

Proof. (1) $(\Rightarrow)$ For each $\Phi: \tau \rightarrow L$, we define

$$
P=\bigvee_{A \in \tau}(\Phi(A) \odot A) .
$$

Since $\tau$ is an Alexandrov topology on $X,(\Phi(A) \odot A) \in \tau$. Thus $P \in \tau$. Then $P=\sqcup \Phi$ from:

$$
\begin{aligned}
e_{\tau}(P, B) & =e_{\tau}\left(\bigvee_{A \in \tau}(\Phi(A) \odot A), B\right) \\
& =\bigwedge_{A \in \tau}\left(\Phi(A) \rightarrow e_{\tau}(A, B)\right) \\
& =e_{\tau}(\sqcup \Phi, B) .
\end{aligned}
$$

For each $\Phi: \tau \rightarrow L$, we define $Q=\bigwedge_{A \in \tau}(\Phi(A) \rightarrow A)$. Since $\tau$ is an Alexandrov topology on $X,(\Phi(A) \rightarrow A) \in \tau$. Thus $Q \in \tau$. Then $Q=\sqcap \Phi$ from:

$$
\begin{aligned}
e_{\tau}(B, Q) & =e_{\tau}\left(B, \bigwedge_{A \in \tau}(\Phi(A) \rightarrow A)\right) \\
& =\bigwedge_{A \in \tau}\left(\Phi(A) \rightarrow e_{\tau}(B, A)\right) \\
& =e_{\tau}(B, \sqcap \Phi) .
\end{aligned}
$$

$(\Rightarrow)$ (T1) For $\Phi(A)=\perp$ for all $A \in \tau, \sqcup \Phi=\bigvee_{A \in \tau}(\Phi(A) \odot$ $A)=\perp_{X} \in \tau$ and $\sqcap \Phi=\bigwedge_{A \in \tau}(\Phi(A) \rightarrow A)=\top_{X} \in \tau$.

(T2) Let $\Phi\left(A_{i}\right)=\top$ for all $\left\{A_{i} \mid i \in \Gamma\right\} \subset \tau$, otherwise $\Phi(A)=\perp$. We have

$$
\begin{aligned}
& \sqcup \Phi=\bigvee_{A \in \tau}(\Phi(A) \odot A)=\bigvee_{i \in \Gamma} A_{i} \in \tau \\
& \sqcap \Phi=\bigwedge_{A \in \tau}(\Phi(A) \rightarrow A)=\bigwedge_{i \in \Gamma} A_{i} \in \tau .
\end{aligned}
$$

(T3) Let $\Phi(A)=\perp$ for $A=B \in \tau$, otherwise $\Phi(A)=\alpha$ if $A \neq B$. We have

$$
\begin{gathered}
\sqcup \Phi=\bigvee_{A \in \tau}(\Phi(A) \odot A)=\alpha \odot B \in \tau \\
\sqcap \Phi=\bigwedge_{A \in \tau}(\Phi(A) \rightarrow A)=\alpha \rightarrow B \in \tau .
\end{gathered}
$$

(2) Let $A^{*} \in \tau^{*}$ for $A \in \tau$. Since $\alpha \odot A^{*}=(\alpha \rightarrow A)^{*}$ and $\alpha \rightarrow A^{*}=(\alpha \odot A)^{*}, \tau^{*}$ is an Alexandrov topology on $X$.

Theorem 2.2. Let $\tau$ be an Alexandrov topology on $X$. Define $\mathcal{I}_{\tau}: L^{X} \rightarrow L^{X}$ as follows:

$$
\mathcal{I}_{\tau}(A)=\bigvee_{B \in \tau}\left(e_{L^{X}}(B, A) \odot B\right)
$$

Then the following properties hold.

(1) $e_{L^{X}}(A, B) \leq e_{L^{X}}\left(\mathcal{I}_{\tau}(A), \mathcal{I}_{\tau}(B)\right)$, for $A, B \in L^{X}$.

(2) $\mathcal{I}_{\tau}(A) \leq A$ for all $A \in L^{X}$.

(3) $\mathcal{I}_{\tau}\left(\mathcal{I}_{\tau}(A)\right)=\mathcal{I}_{\tau}(A)$ for all $A \in L^{X}$.

(4) $\mathcal{I}_{\tau}(\alpha \rightarrow A)=\alpha \rightarrow \mathcal{I}_{\tau}(A)$ for all $\alpha \in L, A \in L^{X}$.

(5) $\mathcal{I}_{\tau}\left(\bigwedge_{i \in \Gamma} A_{i}\right)=\bigwedge_{i \in \Gamma} \mathcal{I}_{\tau}\left(A_{i}\right)$ for all $A_{i} \in L^{X}$.

(6) $\mathcal{I}_{\tau}(\sqcap \Phi)=\sqcap \mathcal{I}_{\tau} \rightarrow(\Phi)$ for each $\Phi: L^{X} \rightarrow L$ where $\mathcal{I}_{\tau} \rightarrow$ $L^{L^{X}} \rightarrow L^{L^{X}}$ defined as $\mathcal{I}_{\tau} \rightarrow(\Phi)(B)=\bigvee_{\mathcal{I}_{\tau}(A)=B} \Phi(A)$.

(7) $I_{\tau}(A)=\bigvee\left\{B \in L^{X} \mid B \leq A, B \in \tau\right\}$.

(8) Define $\tau_{I_{\tau}}=\left\{A \mid A=\mathcal{I}_{\tau}(A)\right\}$. Then $\tau=\tau_{I_{\tau}}$.

(9) There exists a fuzzy preorder $e_{X}: X \times X \rightarrow L$ such that

$$
\mathcal{I}_{\tau}(A)(y)=\bigwedge_{x \in X}\left(e_{X}(x, y) \rightarrow A(x)\right)
$$

Proof. (1) By Lemma $1.2(8,10,14)$, we have

$$
\begin{aligned}
& e_{L^{X}}\left(\mathcal{I}_{\tau}(A), \mathcal{I}_{\tau}(B)\right) \\
& =\bigwedge_{x \in X}\left(\bigvee_{C \in \tau}\left(e_{L^{X}}(C, A) \odot C(x)\right)\right. \\
& \rightarrow \bigvee_{D \in \tau}\left(e_{L^{X}}(D, B) \odot D(x)\right) \\
& \geq \bigwedge_{x \in X} \bigwedge_{C \in \tau}\left(\left(e_{L^{X}}(C, A) \odot C(x)\right)\right. \\
& \left.\rightarrow e_{L^{X}}(C, B) \odot C(x)\right) \\
& \geq \bigwedge_{C \in \tau}\left(\left(e_{L^{X}}(C, A) \rightarrow\left(e_{L^{X}}(C, B)\right)\right.\right. \\
& \geq e_{L^{X}}(A, B)
\end{aligned}
$$

(2) Since $e_{L^{x}}(C, A) \odot C \leq A$ from Lemma $1.2(9), \mathcal{I}_{\tau}(A) \leq$ A.

(3) Since $\mathcal{I}_{\tau}(A) \in \tau$, then

$$
\mathcal{I}_{\tau}\left(\mathcal{I}_{\tau}(A)\right) \geq e_{L^{X}}\left(\mathcal{I}_{\tau}(A), \mathcal{I}_{\tau}(A)\right) \odot \mathcal{I}_{\tau}(A)=\mathcal{I}_{\tau}(A)
$$

By $(2), \mathcal{I}_{\tau}\left(\mathcal{I}_{\tau}(A)\right)=\mathcal{I}_{\tau}(A)$.

(4) Since $\alpha \rightarrow \mathcal{I}_{\tau}(A) \leq \alpha \rightarrow A$ and $\alpha \rightarrow \mathcal{I}_{\tau}(A) \in \tau$,

$$
\begin{aligned}
& \mathcal{I}_{\tau}(\alpha \rightarrow A) \\
& \geq e_{L^{X}}\left(\alpha \rightarrow \mathcal{I}_{\tau}(A), \alpha \rightarrow A\right) \odot\left(\alpha \rightarrow \mathcal{I}_{\tau}(A)\right) \\
& =\alpha \rightarrow \mathcal{I}_{\tau}(A)
\end{aligned}
$$

$$
\begin{aligned}
& \mathcal{I}_{\tau}(\alpha \rightarrow A)=\bigvee_{B \in \tau}\left(e_{L^{X}}(B, \alpha \rightarrow A) \odot B\right) \\
& =\bigvee_{B \in \tau}\left(\left(\alpha \rightarrow e_{L^{X}}(B, A)\right) \odot B\right) \\
& \left.\leq \alpha \rightarrow \bigvee_{B \in \tau}\left(e_{L^{X}}(B, A)\right) \odot B\right)(\text { by Lemma } 1.2(15)) \\
& =\alpha \rightarrow \mathcal{I}_{\tau}(A)
\end{aligned}
$$

(5) By (1), since $\mathcal{I}_{\tau}(A) \leq \mathcal{I}_{\tau}(B)$ for $A \leq B, \mathcal{I}_{\tau}\left(\bigwedge_{i \in \Gamma} A_{i}\right) \leq$ $\bigwedge_{i \in \Gamma} \mathcal{I}_{\tau}\left(A_{i}\right)$. Since $\bigwedge_{i \in \Gamma} \mathcal{I}_{\tau}\left(A_{i}\right) \leq \bigwedge_{i \in \Gamma} A_{i}$ and $\bigwedge_{i \in \Gamma} \mathcal{I}_{\tau}\left(A_{i}\right)$ 
$\in \tau$, we have

$$
\begin{aligned}
& \mathcal{I}_{\tau}\left(\bigwedge_{i \in \Gamma} A_{i}\right) \\
& \left.\geq e_{L^{X}}\left(\bigwedge_{i \in \Gamma} \mathcal{I}_{\tau}\left(A_{i}\right), \bigwedge_{i \in \Gamma} A_{i}\right) \odot \bigwedge_{i \in \Gamma} \mathcal{I}_{\tau}\left(A_{i}\right)\right) \\
& =\bigwedge_{i \in \Gamma} \mathcal{I}_{\tau}\left(A_{i}\right) .
\end{aligned}
$$

(6) For each $\Phi: L^{X} \rightarrow L$, put $Q=\sqcap \mathcal{I}_{\tau}(\Phi)$. Since $\mathcal{I}_{\tau} \rightarrow(\Phi): L^{X} \rightarrow L$ is a map, we have

$$
\sqcap \mathcal{I}_{\tau}^{\rightarrow}(\Phi)=\bigwedge_{C \in L^{X}}\left(\mathcal{I}_{\tau}^{\rightarrow}(\Phi)(C) \rightarrow C\right)
$$

and $Q=\sqcap \mathcal{I}_{\tau}(\Phi)=\mathcal{I}_{\tau}(\sqcap \Phi)$ from:

$$
\begin{aligned}
& e_{L^{X}}(B, Q)=\bigwedge_{C \in L^{X}}\left(\mathcal{I}_{\tau} \rightarrow(\Phi)(C) \rightarrow e_{L^{X}}(B, C)\right. \\
& =e_{L^{X}}\left(B, \bigwedge_{C \in L^{X}}\left(\mathcal{I}_{\tau}^{\rightarrow}(\Phi)(C) \rightarrow C\right)\right) \\
& =e_{L^{X}}\left(B, \bigwedge_{C \in L^{X}}\left(\bigvee_{\mathcal{I}_{\tau}(A)=C} \Phi(A) \rightarrow C\right)\right) \\
& =e_{L^{X}}\left(B, \bigwedge_{A \in L^{X}}\left(\Phi(A) \rightarrow \mathcal{I}_{\tau}(A)\right)\right) \\
& =e_{L^{X}}\left(B, \mathcal{I}_{\tau}\left(\bigwedge_{A \in L^{X}}(\Phi(A) \rightarrow A)\right)\right) \text { (by (4) and (5)) } \\
& =e_{L^{X}}\left(B, \mathcal{I}_{\tau}(\sqcap \Phi)\right) .
\end{aligned}
$$

(7) Put $I(A)=\bigvee\left\{B \in L^{X} \mid B \leq A, B \in \tau\right\}$. Since $I(A) \leq A$ and $I(A) \in \tau$, we have

$\mathcal{I}_{\tau}(A)=\bigvee_{B \in \tau}\left(e_{L^{X}}(B, A) \odot B\right) \geq e_{L^{X}}(I(A), A) \odot I(A)=$ $I(A)$.

Since $\mathcal{I}_{\tau}(A) \leq A$ and $\mathcal{I}_{\tau}(A) \in \tau$, we have $I(A) \geq \mathcal{I}_{\tau}(A)$.

(8) It follows from $A \in \tau$ iff $\mathcal{I}_{\tau}(A)=A$ iff $A \in \tau_{\mathcal{I}_{\tau}}$.

(9) Since $A=\bigwedge_{x \in X}\left(A^{*} \rightarrow \top_{x}^{*}\right)$, by (4) and (5), $\mathcal{I}_{\tau}(A)(y)=$ $\bigwedge_{x \in X}\left(A^{*} \rightarrow \mathcal{I}_{\tau}\left(\top_{x}^{*}\right)(y)\right)=\bigwedge_{x \in X}\left(\mathcal{I}_{\tau}^{*}\left(\top_{x}^{*}\right)(y) \rightarrow A(x)\right)$. Put $e_{X}(x, y)=\mathcal{I}_{\tau}^{*}\left(\top_{x}^{*}\right)(y)$. Then

$$
\begin{gathered}
\mathcal{I}_{\tau}(A)(y)=\bigwedge_{x \in X}\left(e_{X}(x, y) \rightarrow A(x)\right) \\
e_{X}(x, x)=\mathcal{I}_{\tau}^{*}\left(\top_{x}^{*}\right)(x) \geq \top_{x}(x)=\top \\
\bigvee_{y \in X}\left(e_{X}(x, y) \odot e_{X}(y, z)\right) \leq e_{X}(x, z) \\
\text { iff } \bigvee_{y \in X}\left(\mathcal{I}_{\tau}^{*}\left(\top_{x}^{*}\right)(y) \odot \mathcal{I}_{\tau}^{*}\left(\top_{y}^{*}\right)(z)\right) \leq \mathcal{I}_{\tau}^{*}\left(\top_{x}^{*}\right)(z) \\
\text { iff } \bigwedge_{y \in X}\left(\mathcal{I}_{\tau}^{*}\left(\top_{x}^{*}\right)(y) \rightarrow \mathcal{I}_{\tau}\left(\top_{y}^{*}\right)(z)\right) \geq \mathcal{I}_{\tau}\left(\top_{x}^{*}\right)(z) \\
\text { iff } \mathcal{I}_{\tau}\left(\bigwedge_{y \in X}\left(\mathcal{I}_{\tau}^{*}\left(\top_{x}^{*}\right)(y) \rightarrow \top_{y}^{*}\right)\right)(z) \geq \mathcal{I}_{\tau}\left(\top_{x}^{*}\right)(z) \\
\text { iff } \mathcal{I}_{\tau}\left(\mathcal{I}_{\tau}\left(\top_{x}^{*}\right)\right)(z) \geq \mathcal{I}_{\tau}^{*}\left(\top_{x}^{*}\right)(z)
\end{gathered}
$$

Hence $e_{X}$ is a fuzzy preorder.

Theorem 2.3. Let $\tau$ be an Alexandrov topology on $X$. Define $\mathcal{C}_{\tau}: L^{X} \rightarrow L^{X}$ as follows:

$$
\mathcal{C}_{\tau}(A)=\bigwedge_{B \in \tau}\left(e_{L^{x}}(A, B) \rightarrow B\right)
$$

Then the following properties hold.
(1) $e_{L^{X}}(A, B) \leq e_{L^{X}}\left(\mathcal{C}_{\tau}(A), \mathcal{C}_{\tau}(B)\right)$, for all $A, B \in L^{X}$.

(2) $A \leq \mathcal{C}_{\tau}(A)$ for all $A \in L^{X}$.

(3) $\mathcal{C}_{\tau}\left(\mathcal{C}_{\tau}(A)\right)=\mathcal{C}_{\tau}(A)$ for all $A \in L^{X}$.

(4) $\mathcal{C}_{\tau}(\alpha \odot A)=\alpha \odot \mathcal{C}_{\tau}(A)$ for all $\alpha \in L, A \in L^{X}$.

(5) $\mathcal{C}_{\tau}\left(\bigvee_{i \in \Gamma} A_{i}\right)=\bigvee_{i \in \Gamma} \mathcal{C}_{\tau}\left(A_{i}\right)$ for all $A_{i} \in L^{X}$.

(6) $\mathcal{C}_{\tau}(\sqcup \Phi)=\sqcup \mathcal{C}_{\tau}(\Phi)$ for each $\Phi: L^{X} \rightarrow L$ where $\mathcal{C}_{\tau} \rightarrow$ $L^{L^{X}} \rightarrow L^{L^{X}}$ defined as $\mathcal{C}_{\tau}^{\rightarrow}(\Phi)(B)=\bigvee_{\mathcal{C}_{\tau}(A)=B}(\Phi(A))$.

(7) $C_{\tau}(A)=\bigwedge\left\{B \in L^{X} \mid A \leq B, B \in \tau\right\}$.

(8) Define $\tau_{C_{\tau}}=\left\{A \mid A=\mathcal{C}_{\tau}(A)\right\}$. Then $\tau=\tau_{C_{\tau}}$.

(9) $\left(C_{\tau}\left(A^{*}\right)\right)^{*}=I_{\tau^{*}}(A)$ for all $A \in L^{X}$.

(10) There exists a fuzzy preorder $e_{X}: X \times X \rightarrow L$ such that

$$
\begin{aligned}
\mathcal{C}_{\tau}(A)(y) & =\bigvee_{x \in X}\left(e_{X}(x, y) \odot A(x)\right), \\
\mathcal{I}_{\tau^{*}}(A)(y) & =\bigwedge_{x \in X}\left(e_{X}(x, y) \rightarrow A(x)\right) .
\end{aligned}
$$

Proof. (1) By Lemma $1.2(8,10)$, we have

$$
\begin{aligned}
& e_{L^{X}}\left(\mathcal{C}_{\tau}(A), \mathcal{C}_{\tau}(B)\right) \\
& =\bigwedge_{x \in X}\left(\bigwedge_{C \in \tau}\left(e_{L^{X}}(A, C) \rightarrow C(x)\right)\right. \\
& \quad \rightarrow \bigwedge_{D \in \tau}\left(e_{L^{X}}(B, D) \rightarrow D(x)\right) \\
& \geq \bigwedge_{x \in X} \bigwedge_{C \in \tau}\left(\left(e_{L^{X}}(A, C) \rightarrow C(x)\right)\right. \\
& \quad \rightarrow\left(e_{L^{X}}(B, C) \rightarrow C(x)\right) \\
& \geq \bigwedge_{C \in \tau}\left(\left(e_{L^{X}}(B, C) \rightarrow\left(e_{L^{X}}(A, C)\right)\right.\right. \\
& \geq e_{L^{X}}(A, B)
\end{aligned}
$$

(2) Since $e_{L^{X}}(A, B) \odot A \leq B$ iff $A \leq e_{L^{X}}(A, B) \rightarrow B$, then $A \leq \mathcal{C}_{\tau}(A)$.

(3) Since $\mathcal{C}_{\tau}(A) \in \tau$, then $\mathcal{C}_{\tau}\left(\mathcal{C}_{\tau}(A)\right) \leq e_{L^{x}}\left(\mathcal{C}_{\tau}(A), \mathcal{C}_{\tau}(A)\right)$ $\rightarrow \mathcal{C}_{\tau}(A)=\mathcal{C}_{\tau}(A)$. By (2), $\mathcal{C}_{\tau}\left(\mathcal{C}_{\tau}(A)\right)=\mathcal{C}_{\tau}(A)$.

(4) Since $\alpha \odot A \leq \alpha \odot \mathcal{C}_{\tau}(A)$ and $\alpha \odot \mathcal{C}_{\tau}(A) \in \tau$,

$$
\begin{aligned}
\mathcal{C}_{\tau}(\alpha \odot A) & \leq e_{L^{X}}\left(\alpha \odot A, \alpha \odot \mathcal{C}_{\tau}(A)\right) \rightarrow \alpha \odot \mathcal{C}_{\tau}(A) \\
& =\alpha \odot \mathcal{C}_{\tau}(A) .
\end{aligned}
$$

$$
\begin{aligned}
& \mathcal{C}_{\tau}(\alpha \odot A)=\bigwedge_{B \in \tau}\left(e_{L^{X}}(\alpha \odot A, B) \rightarrow B\right) \\
& =\bigwedge_{B \in \tau}\left(\left(\alpha \rightarrow e_{L^{X}}(A, B)\right) \rightarrow B\right) \\
& \geq \bigwedge_{B \in \tau}\left(\alpha \odot\left(e_{L^{X}}(A, B) \rightarrow B\right)\right) \\
& \quad(\text { by Lemmma 1.2(15)) } \\
& \geq \alpha \odot \bigwedge_{B \in \tau}\left(e_{L^{X}}(A, B) \rightarrow B\right) \\
& =\alpha \odot \mathcal{C}_{\tau}(A) .
\end{aligned}
$$

(5) By (1), since $\mathcal{C}_{\tau}(A) \leq \mathcal{C}_{\tau}(B)$ for $A \leq B, \bigvee_{i \in \Gamma} \mathcal{C}_{\tau}\left(A_{i}\right)$ $\leq \mathcal{C}_{\tau}\left(\bigvee_{i \in \Gamma} A_{i}\right)$. Since

$$
\bigvee_{i \in \Gamma} A_{i} \leq \bigvee_{i \in \Gamma} \mathcal{C}_{\tau}\left(A_{i}\right)
$$


and

$$
\bigvee_{i \in \Gamma} \mathcal{C}_{\tau}\left(A_{i}\right) \in \tau
$$

we have

$$
\begin{aligned}
& \mathcal{C}_{\tau}\left(\bigvee_{i \in \Gamma} A_{i}\right) \\
& \leq e_{L^{X}}\left(\bigvee_{i \in \Gamma} A_{i}, \bigvee_{i \in \Gamma} \mathcal{C}_{\tau}\left(A_{i}\right)\right) \rightarrow \bigvee_{i \in \Gamma} \mathcal{C}_{\tau}\left(A_{i}\right) \\
& =\bigvee_{i \in \Gamma} \mathcal{C}_{\tau}\left(A_{i}\right)
\end{aligned}
$$

(6) For each $\Phi: L^{X} \rightarrow L$, put $P=\sqcap \mathcal{C}_{\tau}(\Phi)$. Since $\mathcal{C}_{\tau} \rightarrow(\Phi): L^{X} \rightarrow L$ is a map, we have

$$
\sqcup \mathcal{C}_{\tau}^{\rightarrow}(\Phi)=\bigvee_{C \in \tau}\left(\mathcal{C}_{\tau}^{\rightarrow}(\Phi)(C) \odot C\right)
$$

and $P=\sqcup \mathcal{C}_{\tau} \rightarrow(\Phi)=\mathcal{C}_{\tau}(\sqcup \Phi)$ from:

$$
\begin{aligned}
& e_{L^{X}}(P, B)=\bigwedge_{C \in L^{X}}\left(\mathcal{C}_{\tau} \rightarrow(\Phi)(C) \rightarrow e_{L^{X}}(C, B)\right. \\
& =\bigwedge_{C \in L^{X}} e_{L^{X}}\left(\mathcal{C}_{\tau}^{\rightarrow}(\Phi)(C) \odot C, B\right) \\
& =e_{L^{X}}\left(\bigvee_{C \in L^{X}}\left(\mathcal{C}_{\tau}^{\rightarrow}(\Phi)(C) \odot C\right), B\right) \\
& =e_{L^{X}}\left(\bigvee_{A \in L^{X}}\left(\Phi(A) \odot \mathcal{C}_{\tau}(A)\right), B\right) \\
& =e_{L^{X}}\left(\mathcal{C}_{\tau}\left(\bigvee_{A \in L^{X}}(\Phi(A) \odot A)\right), B\right) \quad(\text { by (4) and (5)) } \\
& =e_{L^{X}}\left(\mathcal{C}_{\tau}(\sqcup \Phi), B\right)
\end{aligned}
$$

(7) Put $C(A)=\bigwedge\left\{B \in L^{X} \mid A \leq B, B \in \tau\right\}$. Since $A \leq C(A)$ and $C(A) \in \tau$, we have

$$
\begin{aligned}
& \mathcal{C}_{\tau}(A)=\bigwedge_{B \in \tau}\left(e_{L^{X}}(A, B) \rightarrow B\right) \\
& \leq e_{L^{X}}(A, C(A)) \rightarrow C(A)=C(A) .
\end{aligned}
$$

Since $A \leq \mathcal{C}_{\tau}(A)$ and $\mathcal{C}_{\tau}(A) \in \tau$, we have $C(A) \leq \mathcal{C}_{\tau}(A)$.

(8) It follows from $A \in \tau$ iff $\mathcal{C}_{\tau}(A)=A$ iff $A \in \tau_{\mathcal{C}_{\tau}}$.

$$
\begin{aligned}
\left(C_{\tau}\left(A^{*}\right)\right)^{*} & =\left(\bigwedge_{B \in \tau}\left(e_{L^{x}}\left(A^{*}, B\right) \rightarrow B\right)\right)^{*} \\
& =\bigvee_{B \in \tau}\left(e_{L^{X}}\left(B^{*}, A\right) \odot B^{*}\right) \\
& =\bigvee_{B^{*} \in \tau^{*}}\left(e_{L^{X}}\left(B^{*}, A\right) \odot B^{*}\right) \\
& =I_{\tau^{*}}(A)
\end{aligned}
$$

(10) Since $A=\bigvee_{x \in X}\left(A \odot \top_{x}\right)$, by (4) and (5), $\mathcal{C}_{\tau}(A)(y)$ $=\bigvee_{x \in X}\left(A \odot \mathcal{C}_{\tau}\left(\top_{x}\right)(y)\right)$. Put $e_{X}(x, y)=\mathcal{C}_{\tau}\left(\top_{x}\right)(y)$. Then

$$
\begin{gathered}
\mathcal{C}_{\tau}(A)(y)=\bigvee_{x \in X}\left(e_{X}(x, y) \odot A(x)\right) \\
e_{X}(x, x)=\mathcal{C}_{\tau}\left(\top_{x}\right)(x) \geq \top_{x}(x)=\top \\
\bigvee_{y \in X}\left(e_{X}(x, y) \odot e_{X}(y, z)\right) \leq e_{X}(x, z) \\
\text { iff } \bigvee_{y \in X}\left(\mathcal{C}_{\tau}\left(\top_{x}\right)(y) \odot \mathcal{C}_{\tau}\left(\top_{y}\right)(z)\right) \leq \mathcal{C}_{\tau}\left(\top_{x}\right)(z) \\
\text { iff } \mathcal{C}_{\tau}\left(\bigvee_{y \in X}\left(\mathcal{C}_{\tau}\left(\top_{x}\right)(y) \odot \top_{y}\right)\right)(z) \leq \mathcal{C}_{\tau}\left(\top_{x}\right)(z) \\
\text { iff } \mathcal{C}_{\tau}\left(\mathcal{C}_{\tau}\left(\top_{x}\right)\right)(z) \leq \mathcal{C}_{\tau}\left(\top_{x}\right)(z)
\end{gathered}
$$

Hence $e_{X}$ is a fuzzy preorder. Since $e_{X}(x, y)=\mathcal{C}_{\tau}\left(\top_{x}\right)(y)=$ $\mathcal{I}_{\tau^{*}}^{*}\left(\top_{x}^{*}\right)(y)$, by Theorem 2.2(9),

$$
\mathcal{I}_{\tau^{*}}(A)(y)=\bigwedge_{x \in X}\left(e_{X}(x, y) \rightarrow A(x)\right) .
$$

Example 2.4. Let $\left(L=[0,1], \odot, \rightarrow,^{*}\right)$ be a complete residuated lattice with a negation defined by

$x \odot y=(x+y-1) \vee 0, \quad x \rightarrow y=(1-x+y) \wedge 1, x^{*}=1-x$.

Let $X=\{x, y, z\}$ be a set and $A_{1}=(1,0.8,0.6), A_{2}=$ $(0.7,1,0.7), A_{3}=(0.5,0.7,1)$.

(1) We define

$$
\begin{aligned}
\tau & =\left\{\bigvee_{i=1}^{3}\left(a_{i} \odot A_{i}\right) \mid A=\left(a_{1}, a_{2}, a_{3}\right) \in L^{X}\right\} \\
& =\left\{e_{X}(A)(y)=\bigvee_{x \in X}\left(e_{X}(x, y) \odot A(x)\right) \mid A \in L^{X}\right\}
\end{aligned}
$$

where

$$
e_{X}=\left(\begin{array}{ccc}
1 & 0.8 & 0.6 \\
0.7 & 1 & 0.7 \\
0.5 & 0.7 & 1
\end{array}\right)
$$

(T1) For $\perp_{X} \in L^{X}, e_{X}\left(\perp_{X}\right)=\perp_{X} \in \tau$. For $\top_{X} \in L^{X}$, $e_{X}\left(\top_{X}\right)=\top_{X} \in \tau$.

(T2) For $e_{X}\left(A_{i}\right) \in \tau$ for each $i \in \Gamma, \bigvee_{i \in \Gamma} e_{X}\left(A_{i}\right)=$ $e_{X}\left(\bigvee_{i \in \Gamma} A_{i}\right) \in \tau$. Moreover, since $e_{X}(A)(x) \geq e_{X}(x, x) \odot$ $A(x)=A(x)$ and $e_{X}\left(e_{X}(A)\right)=e_{X}(A)$,

$$
\bigwedge_{i \in \Gamma} e_{X}\left(A_{i}\right) \leq e_{X}\left(\bigwedge_{i \in \Gamma} e_{X}\left(A_{i}\right)\right) \leq \bigwedge_{i \in \Gamma} e_{X}\left(e_{X}\left(A_{i}\right)\right) .
$$

Hence $\bigwedge_{i \in \Gamma} e_{X}\left(A_{i}\right)=e_{X}\left(\bigwedge_{i \in \Gamma} A_{i}\right) \in \tau$.

(T3) For $e_{X}(A) \in \tau, \alpha \odot e_{X}(A)=e_{X}(\alpha \odot A) \in \tau$.

(T4) Since $\alpha \odot e_{X}\left(\alpha \rightarrow e_{X}(A)\right) \leq e_{X}\left(e_{X}(A)\right)=e_{X}(A)$, we have

$$
\alpha \rightarrow e_{X}(A) \leq e_{X}\left(\alpha \rightarrow e_{X}(A)\right) \leq \alpha \rightarrow e_{X}(A)
$$

Hence, for $e_{X}(A) \in \tau, \alpha \rightarrow e_{X}(A)=e_{X}\left(\alpha \rightarrow e_{X}(A)\right) \in \tau$. Hence $\tau$ is an Alexandrov topology on $X$.

(2) For $B_{1}=(0.7,0.3,0.6), B_{1}=(0.5,0.9,0.3)$, we obtain

$$
\begin{aligned}
& \mathcal{I}_{\tau}\left(B_{1}\right)=(0.5,0.3,0.6), \mathcal{I}_{\tau}\left(B_{2}\right)=(0.5,0.6,0.3), \\
& \mathcal{C}_{\tau}\left(B_{1}\right)=(0.7,0.5,0.6), \mathcal{C}_{\tau}\left(B_{2}\right)=(0.6,0.9,0.6)
\end{aligned}
$$

Let $\Phi: L^{X} \rightarrow L$ as follows

$$
\Phi(B)= \begin{cases}0.9, & \text { if } B=B_{1} \\ 0.8, & \text { if } B=B_{2} \\ 0, & \text { otherwise }\end{cases}
$$




$$
\begin{aligned}
& \sqcap \Phi=\bigwedge_{A \in L^{X}}(\Phi(A) \rightarrow A) \\
& =\left(\Phi\left(B_{1}\right) \rightarrow B_{1}\right) \wedge\left(\Phi\left(B_{2}\right) \rightarrow B_{2}\right) \\
& =(0.9 \rightarrow(0.7,0.3,0.6)) \wedge(0.8 \rightarrow(0.5,0.9,0.3)) \\
& =(0.7,0.4,0.5) \\
& \sqcap \mathcal{I}_{\tau} \rightarrow(\Phi)=\bigwedge_{A \in L^{X}}\left(\Phi(A) \rightarrow \mathcal{I}_{\tau}(A)\right) \\
& =\left(\Phi\left(B_{1}\right) \rightarrow \mathcal{I}_{\tau}\left(B_{1}\right)\right) \wedge\left(\Phi\left(B_{2}\right) \rightarrow \mathcal{I}_{\tau}\left(B_{2}\right)\right) \\
& =(0.9 \rightarrow(0.5,0.3,0.6)) \wedge(0.8 \rightarrow(0.5,0.6,0.3)) \\
& =(0.6,0.4,0.5)
\end{aligned}
$$

Thus, $\mathcal{I}(\sqcap \Phi)=\sqcap \mathcal{I}_{\tau}^{\rightarrow}(\Phi)$.

$$
\begin{aligned}
& \sqcup \Phi=\bigvee_{A \in L^{X}}(\Phi(A) \odot A) \\
& =\left(\Phi\left(B_{1}\right) \odot B_{1}\right) \vee\left(\Phi\left(B_{2}\right) \odot B_{2}\right) \\
& =(0.9 \odot(0.7,0.3,0.6)) \vee(0.8 \odot(0.5,0.9,0.3)) \\
& =(0.6,0.7,0.5) \\
& \sqcup \mathcal{C}_{\tau}(\Phi)=\bigvee_{A \in L^{X}}\left(\Phi(A) \odot \mathcal{C}_{\tau}(A)\right) \\
& =\left(\Phi\left(B_{1}\right) \odot \mathcal{C}_{\tau}\left(B_{1}\right)\right) \vee\left(\Phi\left(B_{2}\right) \odot \mathcal{C}_{\tau}\left(B_{2}\right)\right) \\
& =(0.9 \odot(0.7,0.5,0.6)) \vee(0.8 \odot(0.6,0.9,0.6)) \\
& =(0.6,0.7,0.5)
\end{aligned}
$$

Thus, $\mathcal{C}_{\tau}(\sqcup \Phi)=\sqcup \mathcal{C}_{\tau}(\Phi)$.

(3) We define

$$
\begin{aligned}
& \tau^{*}=\left\{\wedge_{i=1}^{3}\left(a_{i} \rightarrow A_{i}^{*}\right) \mid A=\left(a_{1}, a_{2}, a_{3}\right) \in L^{X}\right\} \\
& =\left\{\bigwedge_{y \in X}\left(A(y) \rightarrow e_{X}^{*}(-, y)\right) \mid A \in L^{X}\right\} \\
& =\left\{\bigwedge_{y \in X}\left(e_{X}(-, y) \rightarrow B(y)\right) \mid B \in L^{X}\right\}
\end{aligned}
$$

For $B_{1}, B_{2}$ and $\Phi$ in (2), we obtain

$$
\begin{aligned}
& \mathcal{I}_{\tau^{*}}\left(B_{1}\right)=\mathcal{C}_{\tau}^{*}\left(B_{1}^{*}\right)=(0.6,0.3,0.6) \\
& \mathcal{I}_{\tau^{*}}\left(B_{2}\right)=\mathcal{C}_{\tau}^{*}\left(B_{2}^{*}\right)=(0.5,0.6,0.3), \\
& \mathcal{C}_{\tau^{*}}\left(B_{1}\right)=(0.7,0.4,0.6), \mathcal{C}_{\tau^{*}}\left(B_{2}\right)=(0.7,0.9,0.6) .
\end{aligned}
$$

Since $\sqcap \Phi=(0.7,0.4,0.5)$ and

$$
\begin{aligned}
& \sqcap \mathcal{I}_{\tau^{*}}(\Phi)=\bigwedge_{A \in L^{X}}\left(\Phi(A) \rightarrow \mathcal{I}_{\tau^{*}}(A)\right) \\
& =\left(\Phi\left(B_{1}\right) \rightarrow \mathcal{I}_{\tau^{*}}\left(B_{1}\right)\right) \wedge\left(\Phi\left(B_{2}\right) \rightarrow \mathcal{I}_{\tau^{*}}\left(B_{2}\right)\right) \\
& =(0.9 \rightarrow(0.6,0.3,0.6)) \wedge(0.8 \rightarrow(0.5,0.6,0.3)) \\
& =(0.7,0.4,0.5)
\end{aligned}
$$

we have $\mathcal{I}_{\tau^{*}}(\sqcap \Phi)=\sqcap \mathcal{I}_{\tau^{*}}(\Phi)$.

Since $\sqcup \Phi=(0.6,0.7,0.5)$ and

$$
\begin{aligned}
& \sqcup \mathcal{C}_{\tau^{*}}^{\rightarrow}(\Phi)=\bigvee_{A \in L^{X}}\left(\Phi(A) \odot \mathcal{C}_{\tau^{*}}(A)\right) \\
& =\left(\Phi\left(B_{1}\right) \odot \mathcal{C}_{\tau^{*}}\left(B_{1}\right)\right) \vee\left(\Phi\left(B_{2}\right) \odot \mathcal{C}_{\tau^{*}}\left(B_{2}\right)\right) \\
& =(0.9 \odot(0.7,0.4,0.6)) \vee(0.8 \odot(0.7,0.9,0.6)) \\
& =(0.6,0.7,0.5)
\end{aligned}
$$

then $\mathcal{C}_{\tau^{*}}(\sqcup \Phi)=\sqcup \mathcal{C}_{\tau^{*}}(\Phi)$.

\section{Conclusions}

The fuzzy complete lattice is defined with join and meet operators on fuzzy partially ordered sets. Alexandrov topologies are the extensions of fuzzy topology and strong topology.

Several properties of join and meet operators induced by Alexandrov topologies in complete residuated lattices have been elicited and proved. In addition, with the concepts of Zhang's completeness, some extensions of interior and closure operators are investigated in the sense of Pawlak's rough set theory on complete residuated lattices. It is expected to find some interesting functorial relationships between Alexandrov topologies and two operators.

\section{Conflict of Interest}

No potential conflict of interest relevant to this article was reported.

\section{References}

[1] Z. Pawlak, "Rough sets," International Journal of Computer \& Information Sciences, vol. 11, no. 5, pp. 341-356, 1982. http://dx.doi.org/10.1007/BF01001956

[2] Z. Pawlak, "Rough probability," Bulletin of the Polish Academy of Sciences Mathematics, vol. 32, pp. 607-615, 1984.

[3] P. Hájek, Metamathematics of Fuzzy Logic. Dordrecht: Kluwer Academic Publisher, 1998.

[4] R. Bĕolhláek, Fuzzy Relational Systems: Foundations and Principles. New York: Kluwer Academic Publishers, 2002.

[5] A. M. Radzikowska and E. E. Kerre, "A comparative study of fuzzy rough sets," Fuzzy Sets and Systems, vol. 126, no. 2, pp. 137-155, 2002. http://dx.doi.org/10.1016/s01650114(01)00032-x

[6] Y. H. She and G. J. Wang, "An axiomatic approach of fuzzy rough sets based on residuated lattices," Computers \& Mathematics with Applications, vol. 58, no. 1, pp. 189-201, 2009. http://dx.doi.org/10.1016/j.camwa.2009. 03.100 
[7] Z. M. Ma and B. Q. Hu, "Topological and lattice structures of L-fuzzy rough sets determined by lower and upper sets," Information Sciences, vol. 218, pp. 194-204, 2013. http://dx.doi.org/10.1016/j.ins.2012.06.029

[8] Q. Y. Zhang and L. Fan, "Continuity in quantitative domains," Fuzzy Sets and Systems, vol. 154, no. 1, pp. 118131, 2005. http://dx.doi.org/10.1016/j.fss.2005.01.007

[9] Q. Zhang, W. Xie, and L. Fan, "Fuzzy complete lattices," Fuzzy Sets and Systems, vol. 160, no. 16, pp. 2275-2291, 2009. http://dx.doi.org/10.1016/j.fss.2008.12.001

[10] Y. C. Kim, "Alexandrov L-topologies," International Journal of Pure and Applied Mathematics, vol. 93, no. 2, pp. 165-179, 2014. http://dx.doi.org/10.12732/ijpam.v93i2.2

[11] Y. C. Kim, "Alexandrov L-topologies and L-join meet approximation operators," International Journal of Pure and Applied Mathematics, vol. 91, no. 1, pp. 113-129, 2014. http://dx.doi.org/10.12732/ijpam.v91i1.12

[12] H. Lai and D. Zhang, "Fuzzy preorder and fuzzy topology," Fuzzy Sets and Systems, vol. 157, no. 14, pp. 1865-1885,
2006. http://dx.doi.org/10.1016/j.fss.2006.02.013

[13] D. Zhang, "An enriched category approach to many valued topology," Fuzzy Sets and Systems, vol. 158, no. 4, pp. 349366, 2007. http://dx.doi.org/10.1016/j.fss.2006.10.001

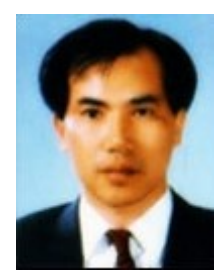

Yong Chan Kim received the B.S., M.S. and $\mathrm{Ph} . \mathrm{D}$. degrees in Mathematics from Yonsei University, Seoul, Korea, in 1982, 1984 and 1991, respectively. He is currently professor of Gangneung-Wonju University. His research interests is a fuzzy topology and fuzzy logic.

E-mail: yck@gwnu.ac.kr

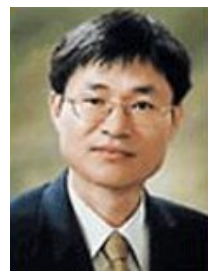

Young Sun Kim received the B.S., M.S. and $\mathrm{Ph} . \mathrm{D}$. degrees in Mathematics from Yonsei University, Seoul, Korea, in 1981, 1985 and 1991, respectively. He is currently Professor of Pai Chai University. His research interests is a fuzzy topology and fuzzy logic.

E-mail: yskim@pcu.ac.kr 